\title{
Prótesis de revisión de cadera en paciente con secuelas de displasia del desarrollo de cadera. Reporte de un caso y revisión de la literatura
}

\author{
Revision hip prosthesis in a patient with sequelae of developmental \\ dysplasia of the hip. Case report and literature review
}

\author{
Darío Esaú Garín Zertuche ${ }^{*}$ Ricardo Enrique Noriega Osornio, ${ }^{\ddagger}$ Christian Michel Ávila Méndez ${ }^{\S}$ \\ ${ }^{*}$ Cirujano Ortopedista. Egresado del Instituto Nacional de Rehabilitación. Diplomado en Artroscopia y Cirugía Articular. \\ Subespecialidad en Reconstrucción Articular. Médico adscrito; $¥$ Médico en Ortopedia y Traumatología. Egresado del Hospital \\ Civil de Culiacán. Fellow en Reconstrucción Articular y Artroscopia; \$Médico en Ortopedia y Traumatología. Egresado del \\ Hospital Centenario Miguel Hidalgo. Fellow en Reconstrucción Articular y Artroscopia. \\ Hospital Ángeles Tijuana. México.
}

\begin{abstract}
Resumen
La displasia del desarrollo de la cadera es una patología que involucra el acetábulo y ocasionalmente afecta el fémur proximal. El objetivo principal al realizar una sustitución protésica de la cadera en este tipo de pacientes es aliviar el dolor y restaurar la función de la cadera. En este caso se trata de una mujer de 26 años con un historial de procedimientos quirúrgicos no satisfactorios, la última cirugía realizada a los 17 años con prótesis total de cadera fallida, requiriendo de cirugía de revisión a los 24 años convirtiéndose en un procedimiento complejo, el cual debe realizarse con una adecuada planeación quirúrgica para realizar una reconstrucción acetabular anatómica y una orientación femoral correcta, con lo implantes idóneos, obteniendo una adecuada biomecánica muscular para la función correcta de la articulación protésica, por lo tanto, este tipo de procedimiento debe ser realizado en manos de un cirujano experto. En la actualidad, la paciente tiene dos años de postoperada con ausencia de dolor y con una función de su extremidad inferior que le ha permitido regresar a realizar sus actividades diarias, así como el inicio de actividad física deportiva, de esta manera mejorando su calidad de vida.
\end{abstract}

Palabras clave: Displasia, cadera, prótesis, reemplazo articular.

\begin{abstract}
Developmental dysplasia of the hip is a pathology that involves the acetabulum and occasionally affects the proximal femur. The primary goal in performing a prosthetic hip replacement in this type of patient is to relieve pain and restore hip function. This case is a 26-year-old female patient with a history of unsatisfactory surgical procedures, the last surgery performed at age 17 with a failed total hip replacement, requiring revision surgery at 24 years of age, making it a complex procedure that must be performed with adequate surgical planning to perform an anatomical acetabular reconstruction and a correct femoral orientation, with the appropriate implants, obtaining an adequate muscular biomechanics for the correct function of the prosthetic joint, therefore, it must be performed by a surgeon who is an expert in this type of procedure. Currently the patient is two years postoperative with absence of pain and with a function of her lower extremity that has allowed her to return to her daily activities, as well as the beginning of sports physical activity, thus improving her quality of life.
\end{abstract}

Keywords: Dysplasia, hip, prosthesis, joint replacement.

Correspondencia:

Dr. Darío Esaú Garín Zertuche

E-mail: dgarinmd@gmail.com

Recibido: 11-05-2021. Aceptado: 09-06-2021.
Citar como: Garín ZDE, Noriega ORE, Ávila MCM. Prótesis de revisión de cadera en paciente con secuelas de displasia del desarrollo de cadera. Reporte de un caso y revisión de la literatura. Orthotips. 2021; 17 (4): 242-245. https://dx.doi.org/10.35366/102223 


\section{Introducción}

La displasia del desarrollo de la cadera es una patología que involucra el acetábulo y ocasionalmente afecta el fémur proximal. La relevancia de realizar un diagnóstico y ofrecer un tratamiento de manera oportuna radica en un buen resultado funcional en estos pacientes. ${ }^{1}$ En los primeros años de vida, la plasticidad de la articulación de la cadera permite tratamientos conservadores para reposicionar el fémur dentro del acetábulo en desarrollo, en caso de fallo al tratamiento conservador, existe el tratamiento quirúrgico que consiste en osteotomías de pelvis y osteotomías femorales. ${ }^{2-4}$

La inestabilidad generada en la cadera por una deformidad en el acetábulo y la cabeza femoral también condicionan anomalías en tejidos blandos, que llevan a una lesión del borde del acetábulo con un riesgo aumentado de lesiones en el cartílago articular y una artrosis secundaria. ${ }^{5}$ Particularmente son pacientes jóvenes que acuden a una consulta con el ortopedista por presentar dolor y limitación funcional de su cadera, se considera que esta patología es causa frecuente de artrosis temprana, lo cual lleva a una sustitución protésica en este grupo de pacientes. ${ }^{6}$

Por lo general, en la cadera displásica se presenta hipoplasia acetabular o femoral, la cual debe ser tratada con implantes diseñados para que se adapten a la forma de la cadera, ${ }^{7,8}$ usualmente implantes de menor tamaño. ${ }^{9}$ La gran mayoría de los

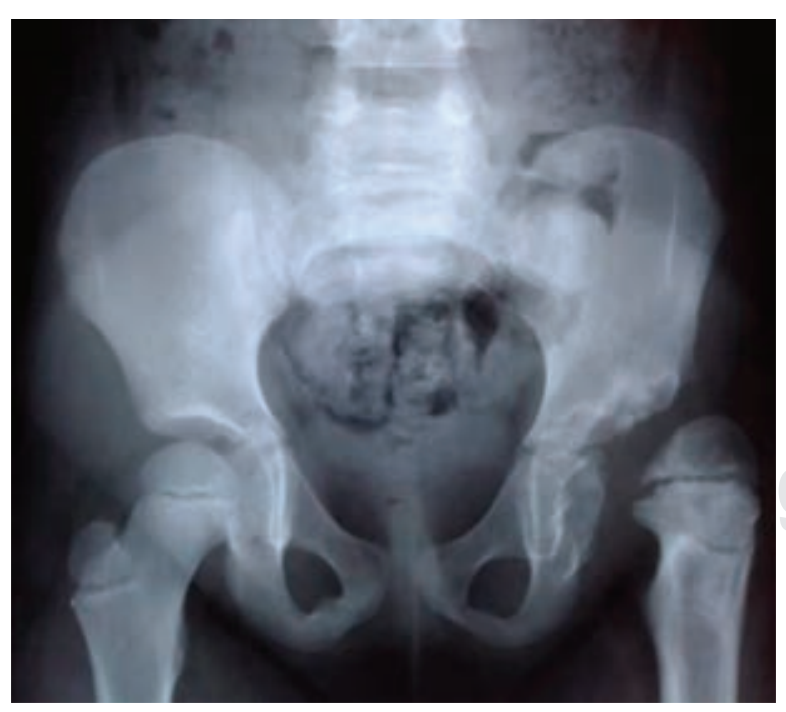

Figura 1: Radiografía de displasia del desarrollo de cadera izquierda en la infancia. Foto del autor.

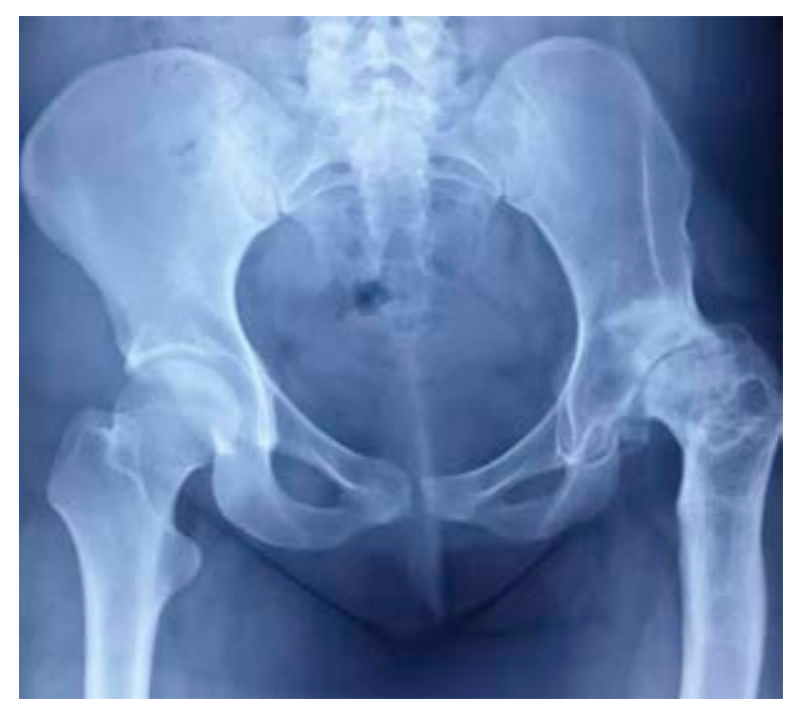

Figura 2: Radiografía de displasia del desarrollo de cadera izquierda en el adulto. Foto del autor.

pacientes tienen historia de múltiples tratamientos tanto conservadores como quirúrgicos, con un mayor grado de dificultad para el reemplazo articular. ${ }^{10}$ Debido a las deformidades anatómicas presentes asociadas en estos pacientes que presentan artrosis, es más frecuente la falla en el reemplazo protésico por mayor grado de dificultad en la técnica quirúrgica. ${ }^{11}$ Existe además una mayor probabilidad de desgaste y aflojamiento aséptico a corto plazo, por una alineación inadecuada en tratamiento protésico primario. ${ }^{12}$

El objetivo principal al realizar una sustitución protésica de la cadera en este tipo de pacientes es aliviar el dolor y restaurar la función de la cadera, y de esta manera restablecer su calidad de vida, permitiéndoles realizar sus actividades diarias, pero con ciertas recomendaciones; en cuanto a los objetivos en el tratamiento es lograr una adecuada colocación y alineación del implante protésico, el cual sea duradero y funcional mediante el equilibrio biomecánico, tanto del componente femoral, como acetabular, permitiendo una adecuada fuerza muscular. ${ }^{13}$

\section{Presentación del caso}

Mujer de 26 años con antecedentes de displasia del desarrollo de la cadera izquierda manejada quirúrgicamente durante la infancia con osteotomía de pelvis y osteotomía desrotadora de fémur, las cuales 
fueron fallidas (Figura 1), presentando secuelas en el desarrollo de su cadera durante su adolescencia con artrosis secundaria (Figura 2), con intenso dolor y limitación a la movilidad, impidiendo sus actividades cotidianas, motivo por el que se le realiza artroplastia total de cadera en clínica privada en el año 2017 a sus 22 años (Figura 3).

En 2018 a sus 23 años acude a mi consulta, refiriendo que continúa con dolor y limitación a la movilidad de la cadera protésica, a la exploración física se encuentra un acortamiento de extremidad inferior izquierda de $3 \mathrm{~cm}$, así como limitación en los arcos de movilidad, se realiza radiografía anteroposterior de pelvis observando subluxación de prótesis total de cadera izquierda, con inadecuada colocación de componentes articulares.

Se decide realizar revisión protésica de cadera bajo consentimiento de paciente en 2019 a sus 24 años, se retira copa acetabular, se realiza osteotomía femoral extendida y se retira vástago, se coloca copa acetabular con $45^{\circ}$ de inclinación acetabular y $10^{\circ}$ de anteversión, en fémur se coloca vástago modular cónico estriado más colocación de tabla de aloinjerto de diáfisis femoral fijándolo mediante cerclaje (Figura 4). En la actualidad, la paciente tiene 26 años, dos años de postoperada con ausencia de dolor y con una función de su extremidad inferior que le ha permitido regresar a realizar sus actividades diarias, así como el inicio de actividad física deportiva, de esta manera mejorando su calidad de vida, se refiere satisfecha con el resultado de su cirugía.

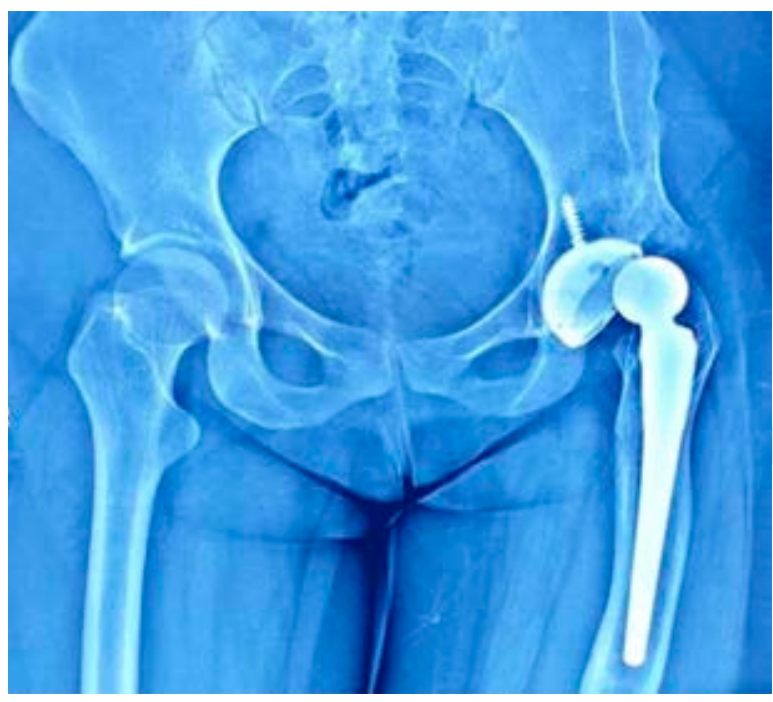

Figura 3: Subluxación de prótesis total de cadera. Foto del autor.
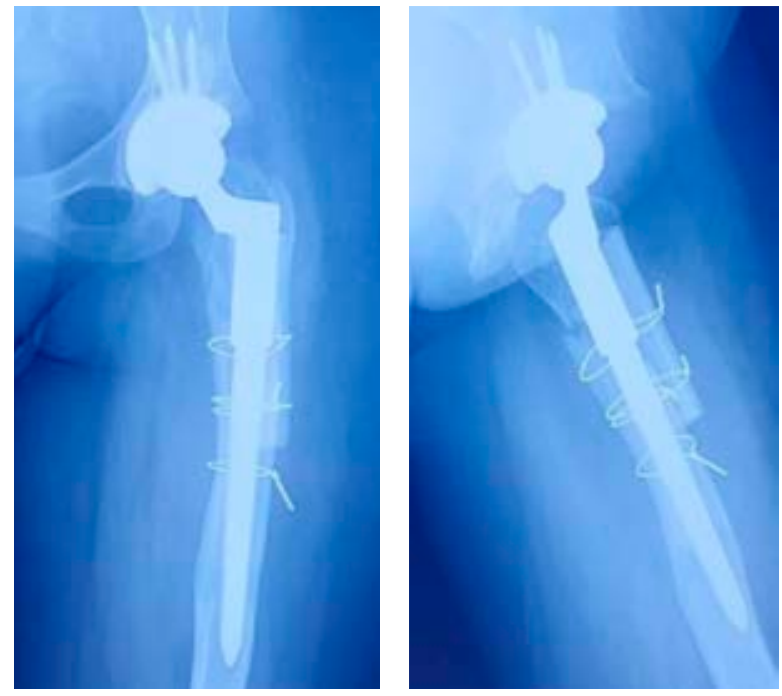

Figura 4: Radiografía postoperatoria de revisión de cadera. Foto del autor.

\section{Discusión}

De acuerdo con la clasificación de Crowe para displasia acetabular, esta paciente presenta un tipo I que corresponde a una migración de la cabeza femoral menor de $50 \%,{ }^{14}$ en este caso el componente acetabular se pudo colocar de manera exitosa, sin necesidad de colocar injerto óseo o aumentos metálicos, ${ }^{15}$ para restituir el centro de rotación de la cadera, sólo requirió de medialización de la copa para proporcionar una adecuada cobertura, ${ }^{16,17}$ el fracaso de la reconstrucción articular protésica se debió a una inadecuada inclinación acetabular y anteversión, motivo por el cual la cadera protésica presentó subluxación, se decide restituir copa acetabular con una inclinación acetabular y anteversión correctas, adecuado centro de rotación de la cadera y un recubrimiento total de la copa. ${ }^{18}$

En lo que respecta al fémur, presenta un defecto proximal que se extiende a la diáfisis, el vástago indicado inicialmente no fue el correcto presentando hundimiento del mismo, ya que estos tipo de fémur presentan su anatomía irregular y son biomecánicamente insuficientes para una fijación metafisaria proximal, se decide retirar vástago realizando una osteotomía lateral femoral extendida, colocando un vástago modular cónico estriado, los cuales son los idóneos para este tipo defectos, ya que presentan una fijación diafisaria distal, donde al menos se requieren de $2 \mathrm{~cm}$ para que sean estables, otorgándoles estabilidad axial y rotacional. ${ }^{19}$ Se colocan tablas de aloinjerto 
diafisarias en defecto femoral proximal con la finalidad de proporcionar soporte estructural disminuyendo el índice de fracturas periprotésicas, así como la no unión de la osteotomía femoral extendida. ${ }^{20}$

\section{Conclusiones}

Para un resultado satisfactorio en este tipo de casos con displasia de cadera, es importante una adecuada planeación quirúrgica para realizar una reconstrucción acetabular anatómica y una orientación femoral correcta, con lo implantes idóneos, obteniendo una adecuada biomecánica muscular para la función correcta de la articulación protésica, regularmente es una cirugía compleja que en su gran mayoría se presenta en pacientes jóvenes y con un historial de procedimientos quirúrgicos fallidos, la cual debe realizarse por un experto.

\section{Referencias}

1. Siddiqi A, White PB, Sloan M, Fox D, Piuzzi NS, Sankar WN, et al. Total hip arthroplasty for developmental dysplasia of hip vs osteoarthritis: a propensity matched pair analysis. Arthroplast Today. 2020; 6 (3): 607-611.e1.

2. Ahmed E, Ganzoury IE, Ayman B. Total hip arthroplasty with subtrochanteric osteotomy in neglected dysplastic hip. Int Orthop. 2015; 39: 27-33.

3. Shi XT, Li CF, Han Y, Song Y, Li SX, Liu JG. Total hip arthroplasty for crowe type IV hip dysplasia: surgical techniques and postoperative complications. Orthop Surg. 2019; 11 (6):966-973.

4. Zhen P, Liu J, Lu H, Chen H, Li X, Zhou S. Developmental hip dysplasia treated by total hip arthroplasty using a cementless Wagner cone stem in young adult patients with a small physique. BMC Musculoskelet Disord. 2017; 18 (1): 192.

5. Wang D, Li LL, Wang HY, Pei FX, Zhou ZK. Long-term results of cementless total hip arthroplasty with subtrochanteric shortening osteotomy in Crowe type IV developmental dysplasia. J Arthroplasty. 2017; 32 (4): 1211-1219.

6. Gala L, Clohisy JC, Beaulé PE. Hip dysplasia in the young adult. J Bone Joint Surg Am. 2016; 98 (1): 63-73.

7. Rollo G, Solarino G, Vicenti G, Picca G, Carrozzo M, Moretti B. Subtrochanteric femoral shortening osteotomy combined with cementless total hip replacement for Crowe type IV developmental dysplasia: a retrospective study. J Orthop Traumatol. 2017; 18 (4): 407-413.

8. Liu S, Zuo J, Li Z, Yang Y, Liu T, Xiao J, et al. Study of three-dimensional morphology of the proximal femur in developmental adult dysplasia of the hip suggests that the onshelf modular prosthesis may not be an ideal choice for patients with Crowe type IV hips. Int Orthop. 2016; 41 (4): 707-713.
9. Schmitz MR, Murtha AS, Clohisy JC; ANCHOR Study Group. Developmental dysplasia of the hip in adolescents and young adults. J Am Acad Orthop Surg. 2020; 28 (3): 91-101.

10. Ding ZC, Zeng WN, Mou P, Liang ZM, Wang D, Zhou ZK. Risk of dislocation after total hip arthroplasty in patients with crowe type iv developmental dysplasia of the hip. Orthop Surg. 2020; 12 (2): 589-600.

11. Zeng WN, Liu JL, Wang FY, Zhang X, Fan HQ, Chen GX, et al. Total hip arthroplasty for patients with Crowe type IV developmental dysplasia of the hip: Ten years results. Int $J$ Surg. 2017; 42: 17-21.

12. Greber EM, Pelt CE, Gililland JM, Anderson MB, Erickson JA, Peters CL. Challenges in total hip arthroplasty in the setting of developmental dysplasia of the hip. J Arthroplasty. 2017; 32 (9S): S38-S44.

13. Bicanic G, Barbaric K, Bohacek I, Aljinovic A, Delimar D. Current concept in dysplastic hip arthroplasty: Techniques for acetabular and femoral reconstruction. World J Orthop. 2014; 5 (4): 412-424.

14. Yang $S$, Cui $Q$. Total hip arthroplasty in developmental dysplasia of the hip: review of anatomy, techniques and outcomes. World J Orthop. 2012; 18: 42-48.

15. Du Y, Fu J, Sun J, Zhang G, Chen J, Ni M et al. Acetabular bone defect in total hip arthroplasty for Crowe II or III developmental dysplasia of the hip: a finite element study. Biomed Res Int. 2020; 2020: 4809013.

16. Zha GC, Sun JY, Guo KJ, Zhao FC, Pang Y, Zheng X. Medial protrusio technique in cementless total hip arthroplasty for developmental dysplasia of the hip: a prospective 6- to 9-year follow-up of 43 consecutive patients. J Arthroplasty. 2016; 31 (8): 1761-1766.

17. Du YQ, Sun JY, Ma HY, Wang S, Ni M, Zhou YG. Leg length balance in total hip arthroplasty for patients with unilateral Crowe type IV developmental dysplasia of the hip. Orthop Surg. 2020; 12 (3): 749-755.

18. Shi XT, Cheng CM, Feng CY, Li CF, Li SX, Liu JG. Crowe type IV hip dysplasia treated by THA comebined with osteotomy to balance functional leg length discrepancy: a prospective observational study. Orthop Surg. 2020; 12 (2): 533-542.

19. Kong $X$, Chai W, Yang M, Ong A, Chen J, Wang $Y$, et al. Modular stem in total hip arthroplasty for patients with trochanter valgus deformity: surgical technique and case series. BMC Musculoskelet Disord. 2020; 21 (1): 124.

20. Lim CT, Amanatullah DF, Huddleston JI 3rd, Hwang KL, Maloney WJ, Goodman SB. Cortical strut allograft support of modular femoral junctions during revision total hip arthroplasty. J Arthroplasty. 2017; 32 (5): 1586-1592.

\section{Conflicto de intereses}

No existe conflicto de intereses por parte de la institución, ni de los médicos involucrados en el reporte de caso. 